\title{
Quasi-free States and Automorphisms of the CCR-Algebra
}

\author{
M. Fannes* \\ Instituut voor Theoretische Natuurkunde, Rijksuniversiteit Groningen, Groningen, The Netherlands
}

\begin{abstract}
We show that any automorphism of the CCR algebra, leaving the quasi-free states globally invariant, is monoparticular.
\end{abstract}

\section{Introduction}

The analogous problem for infinite Fermi systems has been studied in two recent papers. In [2] Hugenholtz and Kadison assume that the gauge invariant quasifree states are globally invariant under the action of an automorphism whereas in [6] Wolfe treats also the situation where all quasi-free states are globally invariant. The conclusion that the automorphism is monoparticular is reached by completely different methods.

The method used in this paper seems again to be quite different from those used in [2] and [6]. The main idea is to introduce an order relation in the set of quasi-free states. We say that $\omega_{1} \lesssim \omega_{2}$ if $\omega_{1} \leqq \gamma \omega_{2}$ for some $\gamma \in \mathbb{R}$; $\lesssim$ defines an ordering because of the exponential character of the quasi-free states.

The main use of $\lesssim$ is to show that adding scalars to the fields $a_{\omega}(\cdot)$ and $a_{\omega}^{*}(\cdot)$ in the representation of a given quasi-free state $\omega$ corresponds to the same kind of transformation for the fields $a_{\omega \circ \alpha}(\cdot)$ and $a_{\omega \circ \alpha}^{*}(\cdot)$, where $\alpha$ denotes the automorphism in question.

\section{Preliminaries [3-5]}

Let $\mathscr{H}$ be a separable (possibly finite dimensional) Hilbert space over $\mathbb{C}$ with inner product $(\cdot \mid \cdot)$ (antilinear in the first component) and $H$ its underlying real Hilbert space. $H=\mathscr{H}$ as a set and the inner product $\langle\cdot \mid \cdot\rangle$ of $H$ is given by

$$
\langle\phi \mid \psi\rangle=\operatorname{Re}(\phi \mid \psi) \quad \phi, \psi \in H .
$$

^ Aspirant van het Belgisch N.F.W.O. On leave from University of Leuven K.U.L. (Belgium). Partially supported by F.O.M. 
Define the bounded linear operator $J$ on $H$ by

$$
J \phi=i \phi
$$

then $J^{2}=-\mathbb{1}$ and $J^{*}=-J$. We need also the nondegenerate symplectic form $\sigma(\cdot \mid \cdot)$ on $H$ given by

$$
\sigma(\phi \mid \psi)=\operatorname{Im}(\phi \mid \psi)=\langle J \phi \mid \psi\rangle \quad \phi, \psi \in H .
$$

The CCR-algebra $\Delta(H, \sigma)$ is the $C^{*}$-algebra obtained by completing the *-algebra $\operatorname{Span}\left\{\delta_{\psi} \mid \psi \in H\right\}$. The elements $\delta_{\psi}$ satisfy the "Weyl"-relations:

$$
\begin{array}{rrr}
\delta_{\psi} \delta_{\phi}=e^{-\frac{i}{2} \sigma(\psi \mid \phi)} \delta_{\psi+\phi} & \phi, \psi \in H, \\
\left(\delta_{\psi}\right)^{*}=\delta_{-\psi} & \psi \in H .
\end{array}
$$

$\Delta(H, \sigma)$ is a simple, non-separable, $C^{*}$-algebra.

For $\phi \in H$ one defines an automorphism $\tau_{\phi}$ of $\Delta(H, \sigma)$ by

$$
\tau_{\phi}(x)=\delta_{\phi} \times \delta_{-\phi}
$$

and one has

$$
\tau_{\phi_{1}} \circ \tau_{\phi_{2}}=\tau_{\phi_{1}+\phi_{2}} \quad \phi_{1}, \phi_{2} \in H .
$$

A $T \in \mathscr{B}(H)$ such that $J^{*} T^{*} J T=T J^{*} T^{*} J=\mathbb{1}$ (equivalently $\sigma(T \phi \mid T \psi)=\sigma(\phi \mid \psi)$ $\phi, \psi \in H$ ) is called a Bogoliubov transformation and it defines an automorphism $\alpha_{T}$ of $\Delta(H, \sigma)$ by:

$$
\alpha_{T}\left(\delta_{\psi}\right)=\delta_{T \psi} \quad \psi \in H .
$$

The quasi-free automorphisms of $\Delta(H, \sigma)$ are those of the form $\tau_{\phi} \circ \alpha_{T}$ where $\phi \in H$ and $T$ is a Bogoliubov transformation.

The quasi-free states $\omega_{(A, \phi)}, A \in Q$ and $\phi \in H$, on $\Delta(H, \sigma)$ are defined by the formula

$$
\omega_{(A, \phi)}\left(\delta_{\psi}\right)=e^{i\langle\phi \mid \psi\rangle} e^{-\frac{1}{4}\langle A \psi \mid \psi\rangle} \quad \psi \in H,
$$

where

$$
Q=\left\{A \in \mathscr{B}(H) \mid A \geqq 0 \text { and } A^{-1} \leqq J^{*} A J\right\} .
$$

As $\omega_{(A, \phi)}=\omega_{(A, 0)}{ }^{\circ} \tau_{J \phi}, \omega_{(A, \phi)}$ is pure iff $\omega_{(A, 0)}$ is pure and this is the case iff $A \geqq 0$ and $A^{-1}=J^{*} A J$. The pure states $\omega_{(A, 0)}$ are also called Fock-states.

\section{A Partial Order Relation on the Quasi-free States}

Definition 3.1. Given $A_{1}, A_{2} \in Q$ and $\phi_{1}, \phi_{2} \in H$, we write

$$
\omega_{\left(A_{1}, \phi_{1}\right)} \precsim \omega_{\left(A_{2}, \phi_{2}\right)}
$$

if there exists a $\gamma \in \mathbb{R}$ such that

$$
\omega_{\left(A_{1}, \phi_{1}\right)} \leqq \gamma \omega_{\left(A_{2}, \phi_{2}\right)} .
$$


Proposition 3.2. i) $\omega_{\left(A_{1}, \phi_{1}\right)} \precsim \omega_{\left(A_{2}, \phi_{2}\right)}$ implies $A_{1} \leqq A_{2}$ and $\phi_{1}-\phi_{2} \in\left[\left(A_{2}-A_{1}\right) H\right]^{-}$; ii) $\lesssim$ defines a partial ordering on the set of quasi-free states.

Proof. i) Let $\gamma \in \mathbb{R}$ be such that

$$
\omega_{\left(A_{1}, \phi_{1}\right)} \leqq \gamma \omega_{\left(A_{2}, \phi_{2}\right)} .
$$

We then also have

$$
\begin{aligned}
\omega_{\left(A_{1}, \phi_{1}-\phi_{2}\right)} & =\omega_{\left(A_{1}, \phi_{1}\right)}{ }^{\circ} \tau_{J^{*} \phi_{2}} \\
& \leqq \gamma \omega_{\left(A_{2}, \phi_{2}\right)}{ }^{\circ} \tau_{J^{*} \phi_{2}} \\
& =\gamma \omega_{\left(A_{2}, 0\right)} .
\end{aligned}
$$

We prove now that $\omega_{\left(A_{1}, \psi\right)} \leqq \gamma \omega_{\left(A_{2}, 0\right)}$ implies

$$
A_{1} \leqq A_{2} \text { and } \psi \in\left[\left(A_{2}-A_{1}\right) H\right]^{-} \text {. }
$$

For any non-zero $\theta \in H$ the function

$$
t \in \mathbb{R} \rightarrow \gamma \omega_{\left(A_{2}, 0\right)}\left(\delta_{t \theta}\right)-\omega_{\left(A_{1}, \psi\right)}\left(\delta_{t \theta}\right)=\gamma e^{-\frac{1}{4} t^{2}\left\langle A_{2} \theta \mid \theta\right\rangle}-e^{i t\langle\psi \mid \theta\rangle} e^{-\frac{1}{4} t^{2}\left\langle A_{1} \theta \mid \theta\right\rangle}
$$

is a continuous function of positive type on the group $(\mathbb{R},+)$. By Bochner's theorem its Fourier transform

$$
k \in \mathbb{R} \rightarrow \frac{\gamma}{\left\langle A_{2} \theta \mid \theta\right\rangle^{\frac{1}{2}}} e^{-\frac{k^{2}}{\left\langle A_{2} \theta \mid \theta\right\rangle}}-\frac{1}{\left\langle A_{1} \theta \mid \theta\right\rangle^{\frac{1}{2}}} e^{-\frac{(k+\langle\psi \mid \theta\rangle)^{2}}{\left\langle A_{1} \theta \mid \theta\right\rangle}}
$$

is positive.

This implies

$$
\begin{array}{lll}
\left\langle A_{1} \theta \mid \theta\right\rangle\left\langle\left\langle A_{2} \theta \mid \theta\right\rangle\right. & \text { if } & \langle\psi \mid \theta\rangle \neq 0 \\
\left\langle A_{1} \theta \mid \theta\right\rangle \leqq\left\langle A_{2} \theta \mid \theta\right\rangle & \text { if } & \langle\psi \mid \theta\rangle=0
\end{array}
$$

and so

$$
A_{1} \leqq A_{2} \text { and } \psi \in\left[\left(A_{2}-A_{1}\right) H\right]^{-} .
$$

ii) Reflexivity and transitivity follow immediately from the definition. Antisymmetry is an immediate consequence of i).

It seems to be quite difficult in general to translate the partial ordening on the states $\omega_{(A, \phi)}$ in terms of $A$ and $\phi$. However, if we restrict to classes of states $\omega_{(A, \phi)}$ where $A$ "belongs" to a fixed "gauge" this can be done.

Let $A \in Q$ and define an inner product $\langle\cdot \mid \cdot\rangle_{A}$ on $H$ by

$$
\langle\phi \mid \psi\rangle_{A}=\langle A \phi \mid \psi\rangle \quad \phi, \psi \in H .
$$

If $J^{*} A=K_{A}|A|_{A}$ is the polar decomposition of $J^{*} A$ with respect to $\langle\cdot \mid \cdot\rangle_{A}$ one shows that $J K_{A}$ defines a Fock state, $\left[K_{A},|A|_{A}\right]=0$ and $|A|_{A} \geqq_{A} \mathbb{1}$. Furthermore $\left\{e^{t K_{A}} \mid t \in \mathbb{R}\right\}$ is a group of Bogoliubov transformations on $H$ and the corresponding group of automorphisms on $\Delta(H, \sigma)$ is called the gauge-group corresponding to $K_{A}$. An operator $B \in Q$ is said to belong to the gauge $K_{A}$ iff $\omega_{(B, 0)}$ is invariant under the corresponding gauge-group. Equivalently $B \in Q$ belongs to the gauge $K_{A}$ iff the polar decomposition of $J^{*} B$ with respect to $\langle\cdot \mid \cdot\rangle_{B}$ is of the form

$$
J^{*} B=K_{A}|B|_{B} \quad[5] .
$$

We first prove two lemma's. 
Lemma 3.3. Suppose that:

i) $\mathscr{H}$ is a complex separable Hilbert space.

ii) $A$ is a (possibly unbounded) self-adjoint linear operator on $\mathscr{H}$ such that $e^{-A} \in \mathscr{B}(\mathscr{H})$.

iii) $\Omega \in \mathscr{H},\|\Omega\|=1$ and $E_{\Omega}$ is the orthogonal projection operator on $\mathbb{C} \Omega$.

Then, there exists a $\gamma \in \mathbb{R}$ such that $E_{\Omega} \leqq \gamma e^{-A}$ iff $\Omega \in \operatorname{Dom}\left(e^{(1 / 2) A}\right)$.

Proof. 1. Suppose that $\Omega \in \operatorname{Dom}\left(e^{(1 / 2) A}\right)$; we show that $\gamma=\left\|e^{(1 / 2) A} \Omega\right\|^{2}$ is a good choice:

For $\psi \in \mathscr{H}$ :

$$
\begin{aligned}
\left\langle E_{\Omega} \psi \mid \psi\right\rangle & =|\langle\psi \mid \Omega\rangle|^{2} \\
& =\left|\left\langle e^{-A / 2} \psi \mid e^{A / 2} \Omega\right\rangle\right|^{2} \\
& \leqq\left\|e^{A / 2} \Omega\right\|^{2}\left\langle e^{-A} \psi \mid \psi\right\rangle \\
& =\gamma\left\langle e^{-A} \psi \mid \psi\right\rangle .
\end{aligned}
$$

2. Conversely suppose that $E_{\Omega} \leqq \gamma e^{-A}$. Define for $\phi \in \operatorname{Dom}\left(e^{(1 / 2) A}\right)$

$$
f(\phi)=\left\langle\Omega \mid e^{(1 / 2) A} \phi\right\rangle .
$$

$f(\cdot)$ is a linear functional and

$$
\begin{aligned}
|f(\phi)|^{2} & =\left|\left\langle\Omega \mid e^{(1 / 2) A} \phi\right\rangle\right|^{2} \\
& =\left\langle e^{(1 / 2) A} \phi \mid E_{\Omega} e^{(1 / 2) A} \phi\right\rangle \\
& \leqq \gamma\left\langle e^{(1 / 2) A} \phi \mid e^{-A} e^{(1 / 2) A} \phi\right\rangle \\
& =\gamma\|\phi\|^{2} .
\end{aligned}
$$

Hence

$$
\left\langle\Omega \mid e^{(1 / 2) A} \phi\right\rangle=\langle\chi \mid \phi\rangle \quad \phi \in \operatorname{Dom}\left(e^{(1 / 2) A}\right)
$$

and so

$$
\Omega \in \operatorname{Dom}\left(e^{(1 / 2) A}\right) .
$$

In the next lemma we compute some estimates for the one boson case: $H=\mathbb{R}^{2}$. If $\{\phi, J \phi\}$ is an orthonormal basis for $H$ we denote by $\delta_{p, q}$ the element

$$
\delta_{p \phi+q J \phi} \quad p, q \in \mathbb{R} .
$$

Lemma 3.4. Consider $\Delta\left(\mathbb{R}^{2}, \sigma\right)$ and, using the same notation as above, the states

$$
\omega_{1}\left(\delta_{p, q}\right)=e^{i(\lambda p+\mu q)} e^{-\frac{1}{4}\left(1+a_{1}\right)\left(p^{2}+q^{2}\right)}
$$

and

$$
\omega_{2}\left(\delta_{p, q}\right)=e^{-\frac{1}{4}\left(1+a_{2}\right)\left(p^{2}+q^{2}\right)},
$$

where $\lambda, \mu \in \mathbb{R}, 0 \leqq a_{1}<a_{2}, a_{1}, a_{2} \in \mathbb{R}$.

Then $\omega_{1} \precsim \omega_{2}$ and the least $\gamma \in \mathbb{R}$ such that $\omega_{1} \leqq \gamma \omega_{2}$ satisfies

$$
\frac{1}{2} \frac{1}{1+a_{2}}\left(a_{2}-a_{1}\right)+\frac{\lambda^{2}+\mu^{2}}{a_{2}-a_{1}} \leqq \ln \gamma \leqq \frac{1}{2}\left(a_{2}-a_{1}\right)+\frac{\lambda^{2}+\mu^{2}}{a_{2}-a_{1}} \text {. }
$$


By Lemma $4.4(*)$ can only be satisfied if $\lambda \rightarrow\left\langle\alpha_{2}\left(A, \lambda \phi+\phi_{0}\right) \mid \psi\right\rangle, \psi \in H$ is affine and so if $\lambda \rightarrow \alpha_{2}\left(A, \lambda \phi+\phi_{0}\right)$ is affine or:

$$
\alpha_{2}\left(A, \lambda \phi+\phi_{0}\right)=\alpha_{2}\left(A, \phi_{0}\right)+\lambda\left\{\alpha_{2}\left(A, \phi+\phi_{0}\right)-\alpha_{2}\left(A, \phi_{0}\right)\right\} .
$$

Substituting (1) in (*), performing the integral and equation both sides we get

$$
\begin{aligned}
& \alpha_{2}\left(A, \phi_{0}\right)=\alpha_{2}\left(A+k \phi \otimes \phi, \phi_{0}\right) \\
& \alpha_{1}(A)+k\left(\alpha_{2}\left(A, \phi+\phi_{0}\right)-\alpha_{2}\left(A, \phi_{0}\right)\right) \otimes\left(\alpha_{2}\left(A, \phi+\phi_{0}\right)-\alpha_{2}\left(A, \phi_{0}\right)\right) \\
& =\alpha_{1}(A+k \phi \otimes \phi) .
\end{aligned}
$$

As the right hand side of (3) is independent of $\phi_{0}$

$$
\alpha_{2}\left(A, \phi+\phi_{0}\right)-\alpha_{2}\left(A, \phi_{0}\right)=\alpha_{2}(A, \phi)-\alpha_{2}(A, 0)
$$

or:

$$
\begin{aligned}
& {\left[\alpha_{2}\left(A, \phi+\phi_{0}\right)-\alpha_{2}(A, 0)\right]} \\
& =\left[\alpha_{2}(A, \phi)-\alpha_{2}(A, 0)\right]+\left[\alpha_{2}\left(A, \phi_{0}\right)-\alpha_{2}(A, 0)\right] \phi, \phi_{0} \in H .
\end{aligned}
$$

Hence the mapping:

$$
T_{A}: \phi \in H \rightarrow \alpha_{2}(A, \phi)-\alpha_{2}(A, 0) \in H
$$

is linear and bounded by Remark 4.2ii).

Let $A, B \in Q$ then $A+B \in Q$. Using Equation (2) for the case $\phi_{0}=0$ and again Remark 4.2ii) we find:

$$
\alpha_{2}(A, 0)=\alpha_{2}(A+B, 0)=\alpha_{2}(B, 0)=\psi_{0} \in H .
$$

Using this (2) reads:

$$
T_{A} \psi=T_{A+k \phi \otimes \phi} \psi \quad \psi \in H .
$$

Using again the same argument as above one has

$$
T_{A}=T_{B}=T_{0} \quad A, B \in Q .
$$

Finally (3) becomes

$$
\alpha_{1}(A)+k T_{0}(\phi \otimes \phi) T_{0}^{*}=\alpha_{1}(A+k \phi \otimes \phi) \quad k \geqq 0 \quad \phi \in H
$$

and this implies that there exists a $C \in \mathscr{B}(\mathscr{H})$ such that

$$
\alpha_{1}(A)=T_{0} A T_{0}^{*}+C(A) .
$$

By the same argument as above one gets

$$
C(A)=C(B) \equiv C_{0} \quad A, B \in Q
$$

and so

$$
\alpha_{1}(A)=T_{0} A T_{0}^{*}+C_{0} \quad A \in Q .
$$

We show that $C_{0}=0$.

First of all $C_{0} \geqq 0$; indeed since $\alpha_{1}(A) \in Q$ one has

$T_{0} A T_{0}^{*}+C_{0} \geqq 0 \quad A \in Q$. 
Observing as in Proposition 3.2i) that $\left(\gamma \omega_{2}-\omega_{1}\right)(\cdot)$ is a function of posit type on the group

$$
\left\{\delta \frac{t \lambda}{\left(\lambda^{2}+\mu^{2}\right)^{\frac{1}{2}}}, \frac{t \mu}{\left(\lambda^{2}+\mu^{2}\right)^{\frac{1}{2}}} \mid t \in \mathbb{R}\right\}
$$

and using again Bochner's theorem we have that

$$
k \in \mathbb{R} \rightarrow \frac{\gamma}{\left(1+a_{2}\right)^{\frac{1}{2}}} e^{-\frac{k^{2}}{\left(1+a_{2}\right)}}-\frac{1}{\left(1+a_{1}\right)^{\frac{1}{2}}} e^{-\frac{\left(k+\left(\lambda^{2}+\mu^{2}\right)^{\frac{1}{2}}\right)^{2}}{1+a_{1}}}
$$

is non-negative. Therefore

$$
\gamma \geqq\left(\frac{1+a_{2}}{1+a_{1}}\right)^{\frac{1}{2}} e^{\frac{\lambda^{2}+\mu^{2}}{a_{2}-a_{1}}}
$$

and so

$$
\ln \gamma \geqq \frac{1}{2} \frac{\left(a_{2}-a_{1}\right)}{1+a_{2}}+\frac{\lambda^{2}+\mu^{2}}{a_{2}-a_{1}} .
$$

Theorem 3.5. Let $A, B \in Q$ belong to the same gauge $K$ and let $\phi, \psi \in H . \omega_{(A, \phi)} \precsim$ $\left.\omega_{(B, \psi)} \leftrightarrow \mathrm{i}\right) A \leqq B$; ii) $B-A$ is trace class on $H$; iii) $\phi-\psi \in \operatorname{Dom}\left(\left(\left.(B-A)\right|_{[(B-A) H]^{-}}\right)^{-\frac{1}{2}}\right)$.

Proof. As there exists a Bogoliubov transformation mapping $J^{*} K$ into $J^{*} J(=\mathbb{1})$ and as the statement of the theorem remains unchanged by performing such a transformation we may suppose $K=J$. We can also assume, without loss of generality, that $\psi=0$.

$(\leftarrow)$ Since $A \in Q$ and $[A, J]=0, \mathbb{1} \leqq A \leqq B$. Consider now the states

$$
\omega_{1}\left(\delta_{\theta}\right)=e^{i\langle\phi \mid \theta\rangle} e^{-\frac{1}{4}\langle\theta \mid \theta\rangle}
$$

and

$$
\omega_{2}\left(\delta_{\theta}\right)=e^{-\frac{1}{4}\langle(\mathbb{1}+B-A) \theta \mid \theta\rangle} .
$$

It is sufficient to show that $\omega_{1} \precsim \omega_{2}$ as by multiplying $\omega_{1}$ and $\omega_{2}$ by the positive type function

$$
\theta \rightarrow e^{-\frac{1}{4}\langle(A-\mathbb{1}) \theta \mid \theta\rangle}
$$

we will have

$$
\omega_{(A, \phi)} \precsim \omega_{(B, 0)} .
$$

By ii) and $[(B-A), J]=0$ there exists an orthonormal basis $\left\{\phi_{1}, J \phi_{1}, \phi_{2}\right.$, $\left.J \phi_{2}, \ldots\right\}$ of $H$ such that

$$
\begin{aligned}
(B-A) \phi_{k} & =\alpha_{k} \phi_{k} \quad \alpha_{k} \in \mathbb{R}^{+} \quad k=1,2, \ldots \\
(B-A) J \phi_{k} & =\alpha_{k} J \phi_{k}
\end{aligned}
$$

and

$$
\sum_{k=1}^{\infty} \alpha_{k}<\infty
$$


Let

$$
\phi=\sum_{k=1}^{\infty}\left(\lambda_{k} \phi_{k}+\mu_{k} J \phi_{k}\right) \quad \lambda_{k}, \mu_{k} \in \mathbb{R}
$$

then by iii):

$$
\begin{aligned}
& \sum_{k=1}^{\infty} \frac{\lambda_{k}^{2}+\mu_{k}^{2}}{\alpha_{k}}<\infty . \\
& \text { For } n=1,2, \ldots \text { let } \\
& H=\left(\bigoplus_{k=1}^{n} H_{k}\right) \oplus H^{n},
\end{aligned}
$$

where

$$
H_{k}=\operatorname{Span}\left\{\phi_{k}, J \phi_{k}\right\} \text {. }
$$

As we have

$$
J H_{k}=H_{k}, J H^{k}=H^{k} \quad k=1,2, \ldots
$$

and as the algebras $\Delta\left(H_{k},\left.\sigma\right|_{H_{k}}\right)$ and $\Delta\left(H^{k},\left.\sigma\right|_{H^{k}}\right)$ are simple we have

$$
\begin{aligned}
\Delta(H, \sigma) & =\left(\bigotimes_{k=1}^{n} \Delta\left(H_{k},\left.\sigma\right|_{H_{k}}\right)\right) \otimes \Delta\left(H^{n},\left.\sigma\right|_{H^{n}}\right) \\
& =\left(\bigotimes_{k=1}^{n} \Delta_{k}\right) \otimes \Delta^{n} .
\end{aligned}
$$

Now

$$
\omega_{1}=\left.\left(\left.\bigotimes_{k=1}^{n} \omega_{1}\right|_{\Delta_{k}}\right) \otimes \omega_{1}\right|_{\Delta^{n}}
$$

and the same holds for $\omega_{2}$.

Using Lemma 3.4 we get that

$$
\omega_{1}\left|\bigotimes_{k=1}^{n} \Delta_{k} \leqq\left(\prod_{k=1}^{n} \gamma_{k}\right) \omega_{2}\right|_{k=1}^{n} \Delta_{k} \quad n=1,2, \ldots
$$

and

$$
0 \leqq \ln \gamma_{k} \leqq \frac{1}{2} \alpha_{k}+\frac{\lambda_{k}^{2}+\mu_{k}^{2}}{\alpha_{k}}
$$

Using $\left(^{*}\right)$ and $(* *)$ we therefore have

$$
\lim _{n \rightarrow \infty} \prod_{k=1}^{n} \gamma_{k}=\gamma<\infty
$$

and so $\omega_{1} \leqq \gamma \omega_{2}$.

$(\rightarrow)$ Let $\gamma \in \mathbb{R}$ be such that $\omega_{(A, \phi)} \leqq \gamma \omega_{(B, 0)}$. Proposition 3.2 gives us condition i) and also $\phi \in[(B-A) H]^{-}$. 
Multiplying $\omega_{(A, \phi)}$ and $\omega_{(B, 0)}$ by the positive type function

$\theta \rightarrow e^{-\frac{1}{2}\langle(\|A\|-A) \theta \mid \theta\rangle}$

we get $\omega_{1} \leqq \gamma \omega_{2}$, where

$$
\omega_{1}\left(\delta_{\theta}\right)=e^{i\langle\phi \mid \phi\rangle} e^{-\frac{1}{4}\langle\|A\| \theta \mid \theta\rangle}
$$

and

$$
\omega_{2}\left(\delta_{\theta}\right)=e^{-\frac{1}{4}\langle(\|A\|+B-A) \theta \mid \theta\rangle} .
$$

Let $\mathscr{D}=\left\{1,2, \ldots \frac{1}{2} \operatorname{dim} H\right\}$ and let $\left(H_{n}\right)_{n \in \mathscr{D}}$ be an increasing sequence of subspaces of $H$, exhausting $H$ and such that

$$
\begin{aligned}
\operatorname{dim} H_{n} & =2 n \quad n \in \mathscr{D} \\
J H_{n} & =H_{n} .
\end{aligned}
$$

For $n \in \mathscr{D}$ define an operator $C_{n}$ as

$$
\left\langle C_{n}, \theta, \chi\right\rangle=\langle(\|A\|+B-A) \theta, \chi\rangle \quad \theta, \chi \in H_{n}
$$

then:

$$
\begin{aligned}
& \mathbb{1}_{H_{n}} \leqq\|A\| \mathbb{1}_{H_{n}} \leqq C_{n} \leqq(\|A\|+\|B\|) \mathbb{1}_{H_{n}} \\
& {\left[C_{n},\left.J\right|_{H_{n}}\right]=0 .}
\end{aligned}
$$

Write also

$$
\phi=\phi_{n}+\phi^{n}, \quad \phi_{n} \in H_{n} \quad \phi^{n}=H \ominus H_{n} .
$$

Restricting the inequality $\omega_{1} \leqq \gamma \omega_{2}$ to the subalgebra Span $\left\{\delta_{\theta} \mid \theta \in H_{n}\right\}$ of $\Delta(H, \sigma)$ we get $\omega_{1}^{n} \leqq \gamma \omega_{2}^{n}$ where $\omega_{1}^{n}$ and $\omega_{2}^{n}$ are the states $\omega_{\left(\|A\| \mathbb{1}_{\left.H_{n}, \phi_{n}\right)}\right.}$ and $\omega_{\left(C_{n}, 0\right)}$ on $\Delta\left(H_{n},\left.\sigma\right|_{H_{n}}\right)$.

Performing the spectral decomposition of $C_{n}$ and using again as in $(\leftarrow)$ Lemma 3.4 we get

$$
\begin{aligned}
\ln \gamma & \geqq \frac{1}{2}\left\|C_{n}\right\|^{-1} \operatorname{Tr}\left(C_{n}-\|A\| \mathbb{1}_{H_{n}}\right)+\left\|\left(C_{n}-\|A\| \mathbb{1}_{H_{n}}\right)^{-\frac{1}{2}} \phi_{n}\right\|^{2} \\
& \geqq 2^{-1}(\|A\|+\|B\|)^{-1} \operatorname{Tr}_{H_{n}}(B-A)+\left\|\left(C_{n}-\|A\| \mathbb{1}_{H_{n}}\right)^{-\frac{1}{2}} \phi_{n}\right\|^{2} .
\end{aligned}
$$

Taking the limit with respect to $n$ we see that

$$
\operatorname{Tr}(B-A)<\infty
$$

and

$\phi \in \operatorname{Dom}\left(\left(\left.(B-A)\right|_{[(B-A) H]^{-}}\right)^{-\frac{1}{2}}\right)$.

\section{Automorphisms Leaving the Quasi-free States Globally Invariant}

From now on we will assume that:

4.1. $\alpha$ is an automorphism of $\Delta(H, \sigma)$ such that its transpose maps the quasifree states on $\Delta(H, \sigma)$ into themselves. We use the following notation:

$$
\omega_{(A, \phi)} \circ \alpha=\omega_{\left(\alpha_{1}(A, \phi), \alpha_{2}(A, \phi)\right)} \quad A \in Q, \phi \in H .
$$


Remark 4.2. If $\alpha$ satisfies 4.1 then

i) $(A, \phi) \rightarrow\left(\alpha_{1}(A, \phi), \alpha_{2}(A, \phi)\right)$ is injective;

ii) $(A, \phi) \rightarrow\left(\alpha_{1}(A, \phi), \alpha_{2}(A, \phi)\right)$ is continuous with respect to weak operator convergence on $H x$ weak convergence on $H$.

Proof. i) is immediate.

ii) Since any $x \in \Delta(H, \sigma)$ can be approximated in norm by a finite linear combination of elements $\delta_{\psi}, \psi \in H$, and since

$$
\omega_{(A, \phi)}\left(\delta_{\psi}\right)=e^{i\langle\phi \mid \psi\rangle} e^{-\frac{1}{4}\langle A \psi \mid \psi\rangle}
$$

it follows that

iff

$$
\omega_{\left(A_{\lambda}, \phi_{\lambda}\right)} \stackrel{w^{*}}{\longrightarrow} \omega_{(A, \phi)}
$$

$A_{\lambda} \stackrel{w}{\rightarrow} A$ and $\quad \phi_{\lambda} \stackrel{w}{\rightarrow} \phi$.

It is then sufficient to remark that $\omega_{\lambda} \circ \alpha \stackrel{w^{*}}{\longrightarrow} \omega \circ \alpha$ is equivalent with $\omega_{\lambda} \stackrel{w^{*}}{\longrightarrow} \omega$.

Lemma 4.3. Let $\alpha$ satisfy 4.1. Then for any $A \in Q$ and $\phi \in H$

$$
\alpha_{1}(A, \phi)=\alpha_{1}(A, 0) \equiv \alpha_{1}(A) \text {. }
$$

Proof. Let $K$ be the gauge corresponding to $A$ and define

$$
C=E_{\phi}+E_{K^{*} \phi}
$$

where $E_{\psi}$ is the projection operator on $\mathbb{R} \psi$. For $\lambda \geqq 0 A+\lambda C \in Q$ and belongs to the same gauge as $A$. Applying Theorem 3.5 we therefore have for $0<\lambda_{1}<\lambda_{2}$

$$
\omega_{(A, \phi)} \precsim \omega_{\left(A+\lambda_{1} C, 0\right)} \precsim \omega_{\left(A+\lambda_{2} C, \phi\right)} .
$$

So, after applying $\alpha$ :

$$
\omega_{\left(\alpha_{1}(A, \phi), \alpha_{2}(A, \phi)\right.} \precsim \omega_{\left(\alpha_{1}\left(A+\lambda_{1} C, 0\right), \alpha_{2}\left(A+\lambda_{1} C, 0\right)\right.} \precsim\left(\omega_{\left(\alpha_{1}\left(A+\lambda_{2} C, \phi\right), \alpha_{2}\left(A+\lambda_{2} C, \phi\right)\right)} .\right.
$$

Then by Proposition 3.2i)

$$
\alpha_{1}(A, \phi) \leqq \alpha_{1}\left(A+\lambda_{1} C, 0\right) \leqq \alpha_{1}\left(A+\lambda_{2} C, \phi\right) .
$$

Taking now the limit $\lambda_{2} \downarrow \lambda_{1} \downarrow 0$ and using Remark 4.2ii) we get

$$
\alpha_{1}(A, \phi) \leqq \alpha_{1}(A, 0) \leqq \alpha_{1}(A, \phi) \text {. }
$$

Lemma 4.4. Suppose that $\lambda \in \mathbb{R} \rightarrow f(\lambda) \in \mathbb{R}$ is continuous and

$$
\pi^{-1 / 2} \int_{\mathbb{R}} d \lambda e^{-\lambda^{2}} e^{i t f(\lambda)}=e^{-(1 / 4) t^{2}} \quad \forall t \in \mathbb{R} .
$$

Then either $f(\lambda)=\lambda$ or $f(\lambda)=-\lambda$.

Proof. One has

$$
e^{-(1 / 4) t^{2}}=\pi^{-1 / 2} \int_{\mathbb{R}} d \lambda e^{-\lambda^{2}} e^{i t \lambda}
$$

and so:

$$
\int_{\mathbb{R}} d \lambda e^{-\lambda^{2}} e^{i t f(\lambda)}=\int_{\mathbb{R}} d \lambda e^{-\lambda^{2}} e^{i t \lambda} \quad \forall t \in \mathbb{R} .
$$


Consider the Hilbert space $\mathscr{H}=\mathscr{L}^{2}\left(\mathbb{R}, e^{-x^{2}} d x\right)$. For $n \in \mathbb{N}$ choose $\alpha_{i} \in \mathbb{C}, t_{i} \in \mathbb{R}$ $i=1 \ldots n$, then by $(*)$ :

$$
\mid \sum_{j=1}^{n} \alpha_{j} e^{i t_{j} \lambda}\left\|^{2}=\right\| \sum_{j=1}^{n} \alpha_{j} e^{i t_{j} f(\lambda)} \|^{2} .
$$

Since $\left\{\lambda \rightarrow e^{i t \lambda} \mid t \in \mathbb{R}\right\}$ is a total set in $\mathscr{H}$, the mapping $U_{f}: \phi \rightarrow \phi \cdot f$ extends to a isometry on $\mathscr{H}$. This implies that $f$ is one to one. By $(*)$ the range of $f$ is the whole of $\mathbb{R}$. Indeed if $] x_{1}, x_{2}[\subset \mathbb{R} \backslash \operatorname{Ran} f$ choose a non-zero positive function $x \rightarrow h(x) \in$ $\mathscr{L}^{1}(\mathbb{R}, d x)$ such that supp $\left.h \subset\right] x_{1}, x_{2}\left[\right.$ and such that $\hat{h} \in \mathscr{L}^{1}(\mathbb{R}, d x)$ (where $\hat{h}$ denotes the Fourier transform of $h$ ). Using $(*)$ one gets:

$$
\begin{aligned}
0 & =\int_{\mathbb{R}} d t \hat{h}(t) \int_{\mathbb{R}} d \lambda e^{-\lambda^{2}} e^{i t f(\lambda)} \\
& =\int_{\mathbb{R}} d t \hat{h}(t) \int_{\mathbb{R}} d \lambda e^{-\lambda^{2}} e^{i t \lambda} \\
& =\int_{\mathbb{R}} d \lambda h(\lambda) e^{-\lambda^{2}} \\
& >0 .
\end{aligned}
$$

This implies that $U_{f}$ is a unitary and so

$$
\int_{\mathbb{R}} d \lambda e^{-\lambda^{2}} g(f(\lambda))=\int_{\mathbb{R}} d \lambda e^{-\lambda^{2}} g(\lambda) \quad g \in \mathscr{L}^{1}\left(\mathbb{R}, e^{-x^{2}} d x\right) .
$$

Clearly $f$ has to be absolutely continuous. One has either

$$
f(+\infty)=\infty \text { or } f(+\infty)=-\infty .
$$

In the first case one gets

or

$$
e^{-\lambda^{2}}=f^{\prime}(\lambda) e^{-f^{2}(\lambda)}
$$

$$
\int_{\lambda}^{+\infty} e^{-s^{2}} d s=\int_{f(\lambda)}^{+\infty} e^{-s^{2}} d s
$$

and so

$$
\lambda=f(\lambda) \text {. }
$$

In the second case one gets $f(\lambda)=-\lambda$.

Theorem 4.5. If $\alpha$ satisfies 4.1 then $\alpha$ is a quasi-free automorphism.

Proof. We use the notation $\phi \otimes \psi, \phi, \psi \in H$ to denote the operator $\chi \in H \rightarrow$ $\langle\phi \mid \chi\rangle \psi \in H$. For $k>0, \phi, \phi_{0} \in H$ and $A \in Q$ one has

$$
(\pi k)^{-1 / 2} \int_{\mathbb{R}} d \lambda e^{-\lambda^{2} / k} \omega_{\left(A, \lambda \phi+\phi_{0}\right)}=\omega_{\left(A+k \phi \otimes \phi, \phi_{0}\right)} .
$$

Applying this on elements $\alpha(x), x \in \Delta(H, \sigma)$ and using Lemma 4.3 we get:

$$
(\pi k)^{-1 / 2} \int_{\mathbb{R}} d \lambda e^{-\lambda^{2} / k} \omega_{\left(\alpha_{1}(A), \alpha_{2}\left(A, \lambda \phi+\phi_{0}\right)\right)}=\omega_{\left(\alpha_{1}(A+k \phi \otimes \phi), \alpha_{2}\left(A+k \phi \otimes \phi, \phi_{0}\right)\right)} .
$$

Let $\psi \in H$ and evaluate both sides on $\delta_{t \psi}, t \in \mathbb{R}$.

$$
\begin{aligned}
& (\pi k)^{-1 / 2} \int_{\mathbb{R}} d \lambda e^{-\lambda^{2} / k} e^{i t\left\langle\alpha_{2}\left(A, \lambda \phi+\phi_{0}\right) \mid \psi\right\rangle} \\
& \left.=e^{i t\left\langle\alpha_{2}\left(A+k \phi \otimes \phi, \phi_{0}\right)\right| \psi}\right\rangle e^{-(1 / 4) t^{2}\left\langle\left(\alpha_{1}(A+k \phi \otimes \phi)-\alpha_{1}(A)\right) \psi \mid \psi\right\rangle} .
\end{aligned}
$$


By Lemma $4.4(*)$ can only be satisfied if $\lambda \rightarrow\left\langle\alpha_{2}\left(A, \lambda \phi+\phi_{0}\right) \mid \psi\right\rangle, \psi \in H$ is affine and so if $\lambda \rightarrow \alpha_{2}\left(A, \lambda \phi+\phi_{0}\right)$ is affine or:

$$
\alpha_{2}\left(A, \lambda \phi+\phi_{0}\right)=\alpha_{2}\left(A, \phi_{0}\right)+\lambda\left\{\alpha_{2}\left(A, \phi+\phi_{0}\right)-\alpha_{2}\left(A, \phi_{0}\right)\right\} .
$$

Substituting (1) in $(*)$, performing the integral and equation both sides we get

$$
\begin{aligned}
& \alpha_{2}\left(A, \phi_{0}\right)=\alpha_{2}\left(A+k \phi \otimes \phi, \phi_{0}\right) \\
& \alpha_{1}(A)+k\left(\alpha_{2}\left(A, \phi+\phi_{0}\right)-\alpha_{2}\left(A, \phi_{0}\right)\right) \otimes\left(\alpha_{2}\left(A, \phi+\phi_{0}\right)-\alpha_{2}\left(A, \phi_{0}\right)\right) \\
& =\alpha_{1}(A+k \phi \otimes \phi) .
\end{aligned}
$$

As the right hand side of (3) is independent of $\phi_{0}$

$$
\alpha_{2}\left(A, \phi+\phi_{0}\right)-\alpha_{2}\left(A, \phi_{0}\right)=\alpha_{2}(A, \phi)-\alpha_{2}(A, 0)
$$

or:

$$
\begin{aligned}
& {\left[\alpha_{2}\left(A, \phi+\phi_{0}\right)-\alpha_{2}(A, 0)\right]} \\
& =\left[\alpha_{2}(A, \phi)-\alpha_{2}(A, 0)\right]+\left[\alpha_{2}\left(A, \phi_{0}\right)-\alpha_{2}(A, 0)\right] \phi, \phi_{0} \in H .
\end{aligned}
$$

Hence the mapping:

$$
T_{A}: \phi \in H \rightarrow \alpha_{2}(A, \phi)-\alpha_{2}(A, 0) \in H
$$

is linear and bounded by Remark $4.2 \mathrm{ii}$ ).

Let $A, B \in Q$ then $A+B \in Q$. Using Equation (2) for the case $\phi_{0}=0$ and again Remark 4.2ii) we find:

$$
\alpha_{2}(A, 0)=\alpha_{2}(A+B, 0)=\alpha_{2}(B, 0)=\psi_{0} \in H .
$$

Using this (2) reads:

$$
T_{A} \psi=T_{A+k \phi \otimes \phi} \psi \quad \psi \in H .
$$

Using again the same argument as above one has

$$
T_{A}=T_{B}=T_{0} \quad A, B \in Q .
$$

Finally (3) becomes

$$
\alpha_{1}(A)+k T_{0}(\phi \otimes \phi) T_{0}^{*}=\alpha_{1}(A+k \phi \otimes \phi) \quad k \geqq 0 \quad \phi \in H
$$

and this implies that there exists a $C \in \mathscr{B}(\mathscr{H})$ such that

$$
\alpha_{1}(A)=T_{0} A T_{0}^{*}+C(A) .
$$

By the same argument as above one gets

$$
C(A)=C(B) \equiv C_{0} \quad A, B \in Q
$$

and so

$$
\alpha_{1}(A)=T_{0} A T_{0}^{*}+C_{0} \quad A \in Q .
$$

We show that $C_{0}=0$.

First of all $C_{0} \geqq 0$; indeed since $\alpha_{1}(A) \in Q$ one has

$T_{0} A T_{0}^{*}+C_{0} \geqq 0 \quad A \in Q$. 
Clearly $C_{0}=C_{0}^{*}$. Suppose that there is a $\theta \in H$ such that $\left\langle C_{0} \theta \mid \theta\right\rangle<0$. It is always possible to choose an $A \in Q$ such that $\left\langle T_{0}^{*} \theta \mid A T_{0}^{*} \theta\right\rangle\left\langle-\frac{1}{2}\left\langle C_{0} \theta \mid \theta\right\rangle\right.$, but this contradicts the positivity of $T_{0} A T_{0}^{*}+C_{0}$.

We prove now that $C_{0} \leqq 0$. Let $\theta \in H$. Since the convex combinations of quasifree states are weakly dense in the set of all states on $\Delta(H, \sigma)$ and since $\alpha$ is an automorphism one has:

$$
\begin{aligned}
1 & =\left\|\delta_{\theta}\right\| \\
& =\left\|\alpha\left(\delta_{\theta}\right)\right\| \\
& =\sup _{0 \leqq \lambda_{i}, \sum_{\substack{A_{i} \in Q_{i} \in H \\
A_{i}}}^{N} \lambda_{i=1}=1 . . N, N \in N}\left|\sum_{i=1}^{N} \lambda_{i} \omega_{\left(A_{l}, \phi_{l}\right)}\left(\alpha\left(\delta_{\theta}\right)\right)\right| \\
& =\sup \left|\sum_{i=1}^{N} \lambda_{i} \omega_{\left(T_{0} A_{i} T_{0}+C_{0}, T_{0} \phi_{i}+\psi_{0}\right)}\left(\delta_{\theta}\right)\right| \\
& \leqq \sup \sum_{i=1}^{N} \lambda_{i} e^{-\frac{1}{4}\left\langle C_{0} \theta \mid \theta\right\rangle} \\
& \leqq e^{-\frac{1}{4}\left\langle C_{0} \theta \mid \theta\right\rangle}
\end{aligned}
$$

and so $C_{0} \leqq 0$.

We conclude that, as for $A \in Q, \phi \in H$

$$
\begin{aligned}
\alpha_{1}(A) & ==T_{0} A T_{0}^{*} \\
\alpha_{2}(A,) & =T_{0} \phi+\psi_{0}
\end{aligned}
$$

$\alpha$ is a quasi-free automorphism.

Acknowledgements. I would like to thank Professor A. Verbeure for suggesting the problem, Professor N.M. Hugenholtz for the kind hospitality at the Rijksuniversiteit Groningen during the final stage of this work and Professor H. Araki for showing me the short proof of Lemma 3.3.1).

\section{References}

1. Guichardet,A.: Symmetric Hilbert spaces and related topics. Lecture Notes in Mathematics 261. Berlin-Heidelberg-New York: Springer 1972

2. Hugenholtz, N.M., Kadison, R.V.: Automorphisms and quasi-free states of the CAR algebra. Commun. math. Phys. 43, 181-197 (1975)

3. Manuceau, J.: $C^{*}$-algèbre de relations de commutation. Ann. Inst. H. Poincaré VIII, 139-161 (1968)

4. Manuceau,J., Sirugue, M., Testard,D., Verbeure,A.: The smallest $C^{*}$-algebra for canonical commutation relations. Commun. math. Phys. 32, 231-243 (1973)

5. Manuceau, J., Verbeure, A.: Quasi-free states of the CCR algebra and Bogoliubov transformations. Commun. math. Phys. 9, 293-302 (1968)

6. Wolfe, J.: Free states and automorphisms of the Clifford algebra. Commun. math. Phys. 45, 53-58 (1975)

Communicated by H. Araki

Received February 28, 1976; in revised form June 14, 1976 